\title{
An Information Theoretic Approach to Optimization of Linear Observations for the Kalman-Bucy Filter
}

\author{
Yoshiki TAKEUCHI and Shinji ESAKI \\ Dept of Information Science, Osaka Univ. of Education \\ 4-698-1 Asahigaoka, Kashiwara City, Osaka 582-8582, Japan
}

\section{E-mail: takeuti@cc.osaka-kyoiku.ac.jp}

\begin{abstract}
we are concerned with a problem of the optimal selection of the gain matrix of a linear observation mechanism for the Kalman-Bucy filter. By introducing an information theoretic constraint, we obtain a gain matrix which maximizes the reduction speed of an weighted estimation error.

In this paper, we are especially concerned with the case where the weighting matrix is not positive but nonnegative definite. By this condition, we can treat an observation with any dimension. This result is more general than the one obtained by one of the authors using a formulation in the optimal transmission framework.
\end{abstract}

\section{Introduction}

The problem of optimization of observations associated with the Kalman-Bucy filter [1] has been studied in many literatures [2]-[5], [8], [9]. In most of them, the problem is formulated as a kind of optimal control problem with a quadratic performance criterion. Logothetis et. al [5], discussed a sensor scheduling problem for linear observations via information theoretic criteria. (See [2], for phisical situations and examples of the sensor scheduling problem.) They treated a discrete-time linear system, and under the constraint that only one sensor can be used at any time, they derived equations for the optimal sensor gains which maximize the mutual information between the signal and the observation. A similar kind of problem is that of sensor allocation. Namely, for a number of sensors, which are simultaneously available at one time, we want to determine a set of gains for the sensors in such a way that the estimation error is minimized. In the view point of the Shannon's information theory, the observation is better when the mutual information between the signal and the observation takes a larger value. However, the mutual information, according to the Shannon's theory, cannot take a larger value than that of the transmission capacity. Also, the set of sensor gains which produces the same mutual information is not unique, and has a certain degree of freedom. In this paper, we consider the optimization of the sensor gains over this degree of freedom. Namely, we obtain the optimal set of gains which maximizes the reduction speed of an weighted estimation error and by which the mutual information takes a preassigned value of the capacity. We are especially concerned with the case where the weighting matrix is not positive but nonnegative definite with a rank which is equal to the dimension of the observation. This condition makes us possible to treat observations with any dimension.

Mathematical symbols, in this paper, are used in the following way. The Euclidean norm is $|\cdot|$. The triplet $(\Omega, \mathscr{F}, P)$ is a complete probability space where $\Omega$ is a sample space with elementary events $\omega, \mathscr{F}$ is a $\sigma$-algebra of subsets of $\Omega$, and $P$ is a probability measure. $\mathrm{E}\{\bullet\}$ denotes the expectation and $E\{\cdot \mid \mathscr{G}\}, \mathscr{G} \subset \mathscr{F}$ the conditional expectation, given $\mathscr{E}$, with respect to $P . \quad \sigma\{\bullet\}$ is the minimal sub- $\sigma$-algebra of $\mathscr{F}$ with respect to which the family of $\mathscr{\mathscr { y }}$-measurable sets or random variables $\{\cdot\}$ is measurable. For an $n \times m$-dimensional matrix $K, \operatorname{im}[K]$ and $\operatorname{ker}[K]$ respectively denotes the image. and kernel spaces of $K$, i.e., $\operatorname{im}[K]=\left\{K \xi \in \boldsymbol{R}^{n} ; \xi \in \boldsymbol{R}^{m}\right\} \quad$ and $\operatorname{ker}[K]=\left\{\xi \in \boldsymbol{R}^{m} ; K \xi=0\right\}$. Let $\boldsymbol{F} \equiv\left\{\mathscr{F}_{t} ; 0 \leq t<\infty\right\}$ be a non-decreasing family of $\sigma$-algebras. A stochastic process $\boldsymbol{x} \equiv\left\{x_{t}(\omega) ; 0 \leq t<\infty\right\}$ is said to be adapted to $\boldsymbol{F}$ or $\boldsymbol{F}$ adapted if $x_{t}(\omega)$ is $\mathscr{F}_{t}$-measurable for all $t \in[0, \infty)$. It is assumed that all random variables and stochastic processes are $\mathscr{F}$-measurable. Unless otherwise stated, stochastic properties are that with respect to $P$.

\section{Problem Formulation}

Let $x_{t}(\omega) \triangleq\left[x_{t}^{1}(\omega) x_{t}^{2}(\omega) \cdots x_{t}^{n}(\omega)\right]^{\prime}$ denote a set of $n$ Gaussian signals. We will assume that $x \equiv\left\{x_{t}(\omega)\right.$; $0 \leq t<\infty\}$ is an $n$-dimensional Gaussian stochastic process given by

$$
x_{t}(\omega)=x_{0}(\omega)+\int_{0}^{t} A(s) x_{s}(\omega) d s+\int_{0}^{t} G(s) d w_{s}(\omega),
$$

where $x_{0}(\omega)$ is a Gaussian random vector and $w \equiv\left\{w_{t}(\omega)\right.$; 
$0 \leq t<\infty\}$ is a $d$-dimensional standard Brownian motion process. Suppose that an $m$-dimensional observation is given by

$$
y_{t}(\omega)=\int_{0}^{t} H(s) x_{s}(\omega) d s+v_{t}(\omega) .
$$

In (2), $y \equiv\left\{y_{t}(\omega) ; 0 \leq t<\infty\right\}$ is an $m$-dimensional observation process, $v \equiv\left\{v_{t}(\omega) ; 0 \leq t<\infty\right\}$ is an $m$-dimensional standard Brownian motion process which is independent of w.

For (1) and (2), the least-squares estimate $\hat{x}_{t}(\omega)$ of $x_{t}(\omega)$ is given by the conditional expectation:

$$
\hat{x}_{t}(\omega) \triangleq E\left\{x_{t}(\omega) \mid \mathscr{Y}_{t}\right\},
$$

where

$$
\mathscr{Y}_{t} \triangleq \sigma\left\{y_{s}(\omega) ; 0 \leq s \leq t\right\} .
$$

The following set of equations to compute the leastsquares estimate is well-known as Kalman-Bucy filter [1]:

$$
\begin{aligned}
& \left\{\begin{array}{l}
d \hat{x}_{t}(\omega) \doteq A \hat{x}_{t}(\omega) d t+Q_{t} H^{\prime}\left\{d y_{t}(\omega)-H \hat{x}_{t}(\omega) d t\right\} \\
\hat{x}_{0}(\omega)=E\left\{x_{0}(\omega)\right\}
\end{array}\right. \\
& \left\{\begin{array}{l}
\frac{d Q_{t}}{d t}=A Q_{t}+Q_{t} A^{\prime}+G G^{\prime}-Q_{t} H^{\prime} H Q_{t} \\
Q_{0}=E\left\{\left[x_{0}(\omega)-\hat{x}_{0}(\omega)\right]\left[x_{0}(\omega)-\hat{x}_{0}(\omega)\right]^{\prime}\right\}
\end{array}\right.
\end{aligned}
$$

where $Q_{t} \triangleq E\left\{\left[x_{t}(\omega)-\hat{x}_{t}(\omega)\right]\left[x_{t}(\omega)-\hat{x}_{t}(\omega)\right]^{\prime}\right\}$ and the time-dependences of $A, G$ and $H$ are abbreviated for simplicity.

Now, let us consider the problem of optimal selection of $H(t)$. In a number of literatures, this problem is formulated as a kind of optimal control problem:

Find $\boldsymbol{H} \equiv\{H(t) ; 0 \leq t<T\}$ by which

$$
J=k_{T}\left(H(T), \operatorname{tr}\left[Q_{T}\right]\right)+\int_{0}^{T} k_{t}\left(H(t), \operatorname{tr}\left[Q_{t}\right]\right) d t,
$$

is minimized for the 'dynamical system' (6).

Unfortunately, however, this is a nonlinear control problem and there arise both theoretical and numerical difficulties to produce few applicable results.

In this paper, by taking into account of the capacity of the observation channel from an information theoretic point of view, we formulate the problem within a local optimization framework to obtain a feasible solution. As we can see from (2), (5) and (6), the estimation error $\operatorname{tr}\left[Q_{t}\right]$ decreases as $H(t) H^{\prime}(t)$ grows. On the other hand, the mutual information between $x$ and $y$ is given by

$$
I_{t}(\boldsymbol{x}, \boldsymbol{y})=\frac{1}{2} \int_{0}^{t} \operatorname{tr}\left[H(s) Q_{s} H^{\prime}(s)\right] d s,
$$

and increases as $H(t) H^{\prime}(t)$ grows. However, according to the Shannon's information theory, we cannot get information larger than the capacity of the transmission channel, i.e., $I_{t}(x, y) \leq C(t)$, where $C(t)$ is the cumulative capacity of the observation (2) up to time $t$. In what follows, we are concerned with the following problem.
[Local Optimization Problem (LOOP)] For any nonnegative definite symmetric matrix $M(t) \in \mathbb{R}^{n \times n}$ with $\operatorname{rank}[M(t)]=m, t \in[0, \infty)$, find $H(t) \in \boldsymbol{R}^{m \times n}$ such that

$$
\operatorname{tr}\left[M(t) \dot{Q}_{t}\right] \rightarrow \min
$$

subject to (6) and

$$
\bar{I}(x, y)=\frac{1}{2} \operatorname{tr}\left[H(t) Q_{t} H^{\prime}(t)\right] \leq c(t),
$$

where $\bar{I}(\boldsymbol{x}, \boldsymbol{y})$ is the mutual information per unit time and $c(t)$ is the capacity of the observation at time $t$.

«Remark 1 》 If $m=n$ and $M(t) \equiv I,(8)$ is reduced to

$$
\operatorname{tr}\left[\dot{Q}_{t}\right] \rightarrow \min .
$$

It should be noted that, even in this case, the non-diagonal elements of $Q_{t}$ are bounded by the diagonal ones. This is easily seen by the Schwartz's inequality

$$
\left|\left[Q_{t}\right]_{i j}\right| \leq \sqrt{\left[Q_{t}\right]_{i i}\left[Q_{t}\right]_{j j}}, \quad 1 \leq i, j \leq n \text {. }
$$

《Remark 2》 It should be noted that the observation of the form:

$$
\tilde{y}_{t}(\omega)=\int_{0}^{t} \tilde{H}(s) x_{s}(\omega) d s+\int_{0}^{t} R(s) d \tilde{v}_{s}(\omega),
$$

is easily converted to (2) when $R_{0}(t) \triangleq R(t) R^{\prime}(t)$ is positive definite for all $t \in[0, \infty)$. In fact, by taking

$$
\begin{gathered}
y_{t}(\omega)=\int_{0}^{t} R_{0}^{-1 / 2}(s) d \tilde{y}_{s}(\omega), \\
H(t)=R_{0}^{-1 / 2}(t) \tilde{H}(t),
\end{gathered}
$$

and

$$
v_{t}(\omega)=\int_{0}^{t} R_{0}^{-1 / 2}(s) R(s) d \tilde{v}_{s}(\omega),
$$

we have (2). Hence, $\tilde{H}(t)$ for (2)' is easily obtained, using solution $H(t)$ for (2), by

$$
\tilde{H}(t)=R_{0}^{1 / 2}(t) H(t) .
$$

《(Remark 3》 It should be noted that for a more general class of problems where the noise covariance is dependent on $\tilde{H}(t)$, i.e., $R \equiv R(t, \tilde{H}(t))$ in (2)', we also have a solution from the one for (2). The solution is easily obtained by the following steps if we can determine $\tilde{H} \in \mathbb{R}^{m \times n}$ such that $R_{0}^{-1 / 2}(t, \tilde{H}) \tilde{H}=H$ for $R_{0} \triangleq R R^{\prime}$ and any $H \in R^{m \times n}$.

(i) Compute optimal $H(t)$ for (2).

(ii) Obtain $\tilde{H}(t)$ for this case as a solution of equation:

$$
R_{0}^{-1 / 2}(t, \tilde{H}(t)) \tilde{H}(t)=H(t) .
$$

\section{Main Results}

In this section, we show a solution to the present problem. First, the condition of optimality is given by the theorem: 
«Theorem 1$\rangle$ Let $p(t) \triangleq 2 c(t)$ and define Lagrangian by

$$
\begin{aligned}
L & \equiv L(t, \lambda) \\
& \triangleq-\operatorname{tr}\left[H Q M Q H^{\prime}\right]+\lambda\left\{\operatorname{tr}\left[H Q H^{\prime}\right]-p\right\} .
\end{aligned}
$$

Then, the condition of optimality for LOOP is given by

$$
\left[M(t) Q_{t}-\lambda(t) I\right] H^{\prime}(t)=0 .
$$

Condition (11) with (9) is described in a useful form as:

《Theorem 2》 Let $\xi^{*}(t)$ be the maximum eigenvalue of $Q_{t}^{1 / 2} M(t) Q_{t}^{1 / 2}$ and $\tilde{\Gamma}(t) \equiv\left[\gamma_{1}(t) \gamma_{2}(t) \cdots \gamma_{\ell}(t)\right]$ be the corresponding set of eigenvectors, where $\ell$ is the multiplicity of $\xi^{*}(t), \ell \leq m$. Then, we have $\lambda(t)=\xi^{*}(t)$ and the optimal gain matrix is given by the following relations.

$$
\begin{gathered}
H^{\prime}(t)=Q_{t}^{-1 / 2} \tilde{\Gamma}(t) K(t), \\
\operatorname{tr}\left[K^{\prime}(t) K(t)\right]=p(t),
\end{gathered}
$$

where $K(t) \in \mathbb{R}^{\ell \times m}$ and $\operatorname{rank}[K(t)]=\ell$.

As we see from (12) and (13), we cannot determine $K(t)$ explicitly from the conditions stated above. In order to guarantee that $\ell$ changes at finite time points and $\xi^{*}(t)$ becomes a mild function, we introduce the following condition.

$$
\dot{\tilde{\Xi}} \triangleq \frac{d}{d t}\left\{\tilde{\Gamma}^{\prime} Q^{1 / 2} M Q^{1 / 2} \tilde{\Gamma}\right\}=\alpha(t) \cdot I
$$

To obtain an applicable expression of (14), we need the following lemma.

《Lemma 1 》 For $M(t) Q_{t}$ and $\xi^{*}(t)$, we have (i) $\hat{\Gamma}(t) \triangleq Q_{t}^{-1 / 2} \tilde{\Gamma}(t)$ is a set of eigenvectors, and

(ii) $\Gamma_{*}^{\prime}(t) \triangleq \tilde{\Gamma}^{\prime}(t) Q_{t}^{1 / 2}$ is a set of left eigenvectors, corresponding to eigenvalue $\xi^{*}(t)$. Furthermore, (iii) $M(t) Q_{t}$ is a simple matrix, i.e., for all eigenvalues, the algebraic and geometric mltiplicities are equal.

«Theorem 3》 Assume that $\dot{M}(t)=F[t, M(t)]$ and $\operatorname{rank}[M(t)]=m$. Then, from (14), we have

$$
\begin{array}{r}
\sum_{j=1}^{m}[K(t)]_{i j}{ }^{2}+s_{i}(t)=\tilde{\alpha}(t), \\
i=1,2, \cdots, \ell,
\end{array}
$$

where

$$
\begin{gathered}
s_{i}(t) \triangleq-\hat{\gamma}_{i}^{\prime}(t)\left\{2 A Q_{t}+G_{0}+\frac{1}{\xi^{*}} Q_{t} F[t, M] Q_{t}\right\} \hat{\gamma}_{i}(t), \\
\tilde{\alpha}(t) \triangleq-\frac{\alpha(t)}{\xi^{*}(t)}, \\
\hat{\Gamma}(t)=\left[\hat{\gamma}_{1}(t) \hat{\gamma}_{2}(t) \cdots \hat{\gamma}_{i}(t)\right],
\end{gathered}
$$

and the time-dependences of $A(t), G_{0}(t) \triangleq G(t) G^{\prime}(t)$,
$M(t)$ and $\xi^{*}(t)$ are abbreviated in (16) for simplicity.

Next, let us examine the relation between the solution given by Theorems 2 and 3, and that of the stationary case obtained in Takeuchi and Sowa [8]. Let

$$
\mathscr{P}(A) \triangleq\left\{H \in \mathbb{R}^{m \times n} ;(H, A) \text { is detectable }\right\} .
$$

Then, the stationary problem for the steady-state value $Q$ of $Q_{t}$ is formulated as follows:

[Stationary Problem] Consider the case where $A(t)=A$, $G(t)=G$ and $p(t)=p$ for all $t \in[0, \infty)$. Find $H \in \mathscr{P}(A)$ such that

$$
\operatorname{tr}[Q] \rightarrow \min .
$$

subject to

$$
\operatorname{tr}\left[H Q H^{\prime}\right] \leq p,
$$

and

$$
A Q+Q A^{\prime}+G G^{\prime}-Q H^{\prime} H Q=0 .
$$

The solution of this problem is given as follows.

[Stationary Solution $]^{[8]}$ Assume that the following conditions are satisfied.

(C-1) $G_{0} \triangleq G G^{\prime}$ is positive definite.

(C-2) $p>2 \operatorname{tr}[A]$.

Then, the optimal stationary solution is given by

$$
\begin{gathered}
A^{\prime} X+X A=-\left(J^{2}-I\right), \\
X Q H^{\prime}=0, \\
Q=\bar{\lambda} J^{-1}\left\{J G_{0} J\right\}^{1 / 2} J^{-1}, \\
\bar{\lambda}=\frac{\operatorname{tr}\left[\left\{J G_{0} J\right\}^{1 / 2}\right]}{p-2 \operatorname{tr}[A]},
\end{gathered}
$$

and

$$
H^{\prime} H=\frac{1}{\bar{\lambda}} J\left\{J G_{0} J\right\}^{-1 / 2} J A+\frac{1}{\bar{\lambda}} A^{\prime} J\left\{J G_{0} J\right\}^{-1 / 2} J+\frac{1}{\bar{\lambda}^{2}} J^{2},
$$

where

$$
J^{2} \triangleq \bar{\lambda}^{2} Q^{-1} G_{0} Q^{-1}
$$

«Theorem 4» Assume that $A(t)=A, G(t)=G$ and $p(t)=p$ for all $t \in[0, \infty)$, and that the conditions (C-1)(C-2) are satisfied. Let $Q=\bar{Q}$ and $H=\bar{H}$ be the solution of the stationary problem. Also, since $\operatorname{rank}[X]=n-m$, let $X=\bar{U} \bar{\Phi} \bar{U}^{\prime}, \quad$ where $\quad \bar{\Phi}=\operatorname{diag}\left(\bar{\phi}_{m+1}, \bar{\phi}_{m+2}, \cdots, \bar{\phi}_{n}\right) \quad$ and $\bar{U}^{\prime} \bar{U}=I$. Let us determine $M(t)$ for LOOP by

$$
M(t) \equiv \bar{Q}^{-1}-\bar{U} \bar{U}^{\prime} \bar{Q}^{-1} \bar{U} \bar{U}^{\prime} .
$$

If $\ell=m$ and $\operatorname{im}\left[Q_{t} H^{\prime}(t)\right]=\operatorname{im}\left[\bar{Q} \bar{H}^{\prime}\right]$ at any time $t$ for a solution of LOOP, then we have $\xi^{*}(t) \rightarrow 1$, $Q_{t} H^{\prime}(t) \rightarrow \bar{Q} \bar{H}^{\prime}, \quad H^{\prime}(t) \rightarrow \bar{H}^{\prime}$ and $Q_{t} \rightarrow \bar{Q}$ as $t \rightarrow \infty$. 
〈Remark 4)〉 It should be noted that the condition (9) and/or (13) are violated in the non-stationary phase if we use the stationary optimal gain matrix $H(t) \equiv \bar{H}$. By this selection, for a solution of the Riccati differential equation:

$$
\dot{Q}_{t}=A Q_{t}+Q_{t} A^{\prime}+G G^{\prime}-Q_{t} \bar{H}^{\prime} \bar{H} Q_{t},
$$

we also have the asymptotic property:

$$
\lim _{t \rightarrow \infty} Q_{t}=\bar{Q} \text {. }
$$

However, for any initial value $Q_{0}$ of $Q_{t}$ such that $Q_{0}>\bar{Q}$ and $\xi^{*}(0)>1$, we have $\operatorname{tr}\left[\bar{H} Q_{t} \bar{H}^{\prime}\right]>\operatorname{tr}\left[\bar{H} \bar{Q} \bar{H}^{\prime}\right]$ $=p(t)$ for the solution of (29) in the transient phase.

\section{Proofs of Theorems}

In this section, we will give proofs of the theorems presented in the previous section. First let us show Theorem 1.

(Proof of Theorem 1) First, let us note that from (6), we have

$$
\begin{aligned}
\operatorname{tr}[M \dot{Q}] & =2 \operatorname{tr}[M A Q]+\operatorname{tr}\left[M G_{0}\right] \\
& -\operatorname{tr}\left[H Q M Q H^{\prime}\right] .
\end{aligned}
$$

Since the first two terms in the right-hand side of (30) do not depend on $H$, the value of the left-hand side is minimized when $-\operatorname{tr}\left[H Q M Q H^{\prime}\right]$ is minimized. Note that for any symmetric matrix $X \in \mathbb{R}^{n \times n}$, we have

$$
\frac{\partial}{\partial H} \operatorname{tr}\left[H X H^{\prime}\right]=2 H X \text {. }
$$

Then, from (10), the condition for optimality is

$$
\frac{\partial L}{\partial H}=-2 H(t)\left[Q_{t} M(t)-\lambda(t) I\right] Q_{t}=0,
$$

and which implies (11) since $Q_{t}>0$.

Now, let us proceed to the proof of Theorem 2 .

(Proof of Theorem 2) First, let us note that substitution of (11) into (10) yields

$$
L(t, \lambda)=-\lambda(t) p(t)
$$

Hence, $L(t, \lambda)$ is minimum when $\lambda(t)$ which satisfies (11) takes a maximum value. From (11), we have

$$
\left[M Q^{1 / 2}-\lambda Q^{-1 / 2}\right] Q^{1 / 2} H^{\prime}=0
$$

which implies

$$
\left[Q^{1 / 2} M Q^{1 / 2}-\lambda I\right] Q^{1 / 2} H^{\prime}=0 .
$$

Note that for $\lambda(t)>\xi^{*}(t)$, (35) does not hold since the matrix in the blanket of (35) becomes, then, negative definite and $H(t) \neq 0$. Then, it follows from (33) that $L(t, \lambda)$ becomes minimum when $\lambda(t)=\xi^{*}(t)$, and each column vector of $Q^{1 / 2} H^{\prime}$ is a linear combination of column vectors of $\tilde{\Gamma}(t)$. This is also seen by the fact that (35) implies

$$
Q^{1 / 2} M Q^{1 / 2} \cdot Q^{1 / 2} H^{\prime}=\lambda Q^{1 / 2} H^{\prime},
$$

i.e., the column vectors of $Q^{1 / 2} H^{\prime}$ are eigenvectors of $Q^{1 / 2} M Q^{1 / 2}$ corresponding to the eigenvalue $\lambda(t)=\xi^{*}(t)$. Hence, we can write

$$
Q_{t}^{1 / 2} H^{\prime}(t)=\tilde{\Gamma}(t) K(t),
$$

which implies (12). Now, it can be seen from (12) that

$$
\begin{aligned}
& \operatorname{tr}\left[H Q H^{\prime}\right]=\operatorname{tr}\left[K^{\prime} \tilde{\Gamma}^{\prime} Q^{-1 / 2} Q Q^{-1 / 2} \tilde{\Gamma} K\right] \\
& =\operatorname{tr}\left[K^{\prime} \tilde{\Gamma}^{\prime} \tilde{\Gamma} K\right]=\operatorname{tr}\left[K^{\prime} K\right] .
\end{aligned}
$$

Substitutions of (38) and $p(t) \triangleq 2 c(t)$ into (9) yield (13).

(Proof of Lemma 1) The proof is rather trivial. Since column vectors of $\tilde{\Gamma}(t)$ are eigenvectors of $Q^{1 / 2} M Q^{1 / 2}$, we have

$$
Q^{1 / 2} M Q^{1 / 2} \cdot \tilde{\Gamma}=\xi^{*} \tilde{\Gamma}
$$

Hence,

$$
\begin{aligned}
M Q \cdot \hat{\Gamma} & =M Q Q^{-1 / 2} \tilde{\Gamma} \\
& =Q^{-1 / 2} Q^{1 / 2} M Q^{1 / 2} \tilde{\Gamma} \\
& =Q^{-1 / 2} \xi^{*} \tilde{\Gamma}=\xi^{*} \hat{\Gamma} .
\end{aligned}
$$

Thus, we have (i). Similarly,

$$
\begin{aligned}
\Gamma_{*}^{\prime} \cdot M Q & =\tilde{\Gamma}^{\prime} Q^{1 / 2} M Q \\
& =\tilde{\Gamma}^{\prime} Q^{1 / 2} M Q^{1 / 2} \cdot Q^{1 / 2} \\
& =\xi^{*} \tilde{\Gamma}^{\prime} Q^{1 / 2}=\xi^{*} \Gamma_{*}^{\prime},
\end{aligned}
$$

which implies (ii). Finally, since

$$
\begin{aligned}
\operatorname{det}\left|M Q-\xi^{*} I\right| & =\operatorname{det}\left|Q^{-1 / 2}\left[Q^{1 / 2} M Q^{1 / 2}-\xi^{*} I\right] Q^{1 / 2}\right| \\
& =\operatorname{det}\left|Q^{1 / 2} M Q^{1 / 2}-\xi^{*} I\right|,
\end{aligned}
$$

and

$$
\begin{aligned}
\operatorname{rank}\left|M Q-\xi^{*} I\right| & =\operatorname{rank}\left|Q^{-1 / 2}\left[Q^{1 / 2} M Q^{1 / 2}-\xi^{*} I\right] Q^{1 / 2}\right| \\
& =\operatorname{rank}\left|Q^{1 / 2} M Q^{1 / 2}-\xi^{*} I\right|,
\end{aligned}
$$

we have (iii). This completes the proof.

(Proof of Theorem 3) First, let us note that

$$
\tilde{\Gamma}^{\prime} Q^{1 / 2} M Q^{1 / 2} \tilde{\Gamma}=\Gamma_{*}^{\prime} M Q \hat{\Gamma} .
$$

Then, it follows that

$$
\begin{aligned}
& \frac{d}{d t}\left\{\tilde{\Gamma}^{\prime} Q^{1 / 2} M Q^{1 / 2} \tilde{\Gamma}\right\}=\frac{d}{d t}\left\{\Gamma_{*}^{\prime} M Q \hat{\Gamma}\right\} \\
& \quad=\dot{\Gamma}_{*}^{\prime} M Q \hat{\Gamma}+\Gamma_{*}^{\prime} M Q \dot{\hat{\Gamma}}+\Gamma_{*}^{\prime}(M \dot{Q}+\dot{M} Q) \hat{\Gamma} .
\end{aligned}
$$

By (40) and (41), we have

$$
\dot{\Gamma}_{*}^{\prime} M Q \hat{\Gamma}=\xi^{*} \dot{\Gamma}_{*}^{\prime} \hat{\Gamma},
$$

and

$$
\Gamma_{*}^{\prime} M Q \dot{\hat{\Gamma}}=\xi^{*} \Gamma_{*}^{\prime} \dot{\hat{\Gamma}} .
$$


Hence,

$$
\begin{aligned}
& \dot{\Gamma}_{*}^{\prime} M Q \hat{\Gamma}+\Gamma_{*}^{\prime} M Q \dot{\hat{\Gamma}}=\xi^{*}\left(\dot{\Gamma}_{*}^{\prime} \hat{\Gamma}+\Gamma_{*}^{\prime} \dot{\hat{\Gamma}}\right) \\
& \quad=\xi^{*} \frac{d}{d t}\left(\Gamma_{*}^{\prime} \hat{\Gamma}\right)=0,
\end{aligned}
$$

where the last equality follows from $\Gamma_{0}^{\prime} \hat{\Gamma}=I$ which is obvious by Lemma 1. For the third term of (45), we have

\section{$\Gamma_{*}^{\prime} M \dot{Q} \hat{\Gamma}$}

$$
\begin{aligned}
& =\Gamma_{*}^{\prime} M\left\{A Q+Q A^{\prime}+G_{0}\right\} \hat{\Gamma} \\
& -\Gamma_{*}^{\prime} M Q H^{\prime} H Q \hat{\Gamma} .
\end{aligned}
$$

It can be seen from (41) that

$$
\begin{aligned}
\Gamma_{*}^{\prime} M & \left.M A Q+Q A^{\prime}+G_{0}\right\} \hat{\Gamma} \\
= & \Gamma_{*}^{\prime} M Q Q^{-1}\left\{A Q+Q A^{\prime}+G_{0}\right\} \hat{\Gamma} \\
= & \xi^{*} \Gamma_{*}^{\prime} Q^{-1}\left\{A Q+Q A^{\prime}+G_{0}\right\} \hat{\Gamma} \\
= & \xi^{*} \hat{\Gamma}^{\prime}\left\{A Q+Q A^{\prime}+G_{0}\right\} \hat{\Gamma} .
\end{aligned}
$$

and that

$$
\begin{aligned}
\Gamma^{\prime} & M Q H^{\prime} H Q \hat{\Gamma} \\
& =\xi^{*} \Gamma^{\prime} Q^{-1 / 2} \tilde{\Gamma} K K^{\prime} \tilde{\Gamma}^{\prime} Q^{-1 / 2} Q \hat{\Gamma} \\
& =\xi^{*} \tilde{\Gamma}^{\prime} \tilde{\Gamma} K K^{\prime} \tilde{\Gamma} \tilde{\Gamma}=\xi^{*} K K^{\prime},
\end{aligned}
$$

where (12) is also used for the first equality. Finally, we see that

$$
\begin{gathered}
\Gamma_{*}^{\prime} \dot{M} Q \hat{\Gamma}=\Gamma_{*}^{\prime} F[t, M] Q \hat{\Gamma} \\
\quad=\Gamma_{*}^{\prime} Q^{-1} Q F[t, M] Q \hat{\Gamma} \\
\quad=\hat{\Gamma}^{\prime} Q F[t, M] Q \hat{\Gamma} .
\end{gathered}
$$

Then, from (45) and (48)-(52), we have

$$
\begin{aligned}
& \frac{d}{d t}\left\{\tilde{\Gamma}^{\prime} Q^{1 / 2} M Q^{1 / 2} \tilde{\Gamma}\right\} \\
& \quad=\xi^{*} \hat{\Gamma}^{\prime}\left\{A Q+Q A^{\prime}+G_{0}\right\} \hat{\Gamma}+\hat{\Gamma}^{\prime} Q F[t, M] Q \hat{\Gamma} \\
& \quad-\xi^{*} K K^{\prime} .
\end{aligned}
$$

By (53) and (14), we have (15) and (16) from the relations for the diagonal elements.

(Proof of Theorem 4) Since $\operatorname{im}\left[Q_{t} H^{\prime}(t)\right]=\operatorname{im}\left[\bar{Q} \bar{H}^{\prime}\right]$, we can write

$$
Q_{t} H^{\prime}(t)=\sqrt{\xi^{*}(t)} \bar{Q} \bar{H}^{\prime} L(t), \quad L(t) \in \boldsymbol{R}^{m \times m},
$$

where $\operatorname{rank}[L(t)]=m$. From (54) and (11), we have

$$
\sqrt{\xi^{*}(t)} H(t)=L^{\prime}(t) \bar{H},
$$

since $\lambda(t)=\xi^{*}(t)$ and

$$
\begin{aligned}
& M(t) Q_{t} H^{\prime}(t) \\
& =\left[\bar{Q}^{-1}-\bar{U} \bar{U}^{\prime} \bar{Q}^{-1} \bar{U} \bar{U}^{\prime}\right] \sqrt{\xi^{*}(t)} \bar{Q} \bar{H}^{\prime} L(t) \\
& =\sqrt{\xi^{*}(t)} \bar{H}^{\prime} L(t),
\end{aligned}
$$

where the last equality follows from (23) and $X=\bar{U} \bar{\Phi} \bar{U}^{\prime}$. Also, from (54) and (55), we must have

$$
\operatorname{tr}\left[H(t) Q_{t} H^{\prime}(t)\right]=\operatorname{tr}\left[L^{\prime}(t) \bar{H} \bar{Q} \bar{H}^{\prime} L(t)\right]=p .
$$

First, let us note that conditions (11) and (57) are satisfied with $L(t)=I$ since $\operatorname{tr}\left[\bar{H} \bar{Q} \bar{H}^{\prime}\right]=p$. However, we do not have (14) for this selection of $L(t)$. Let $\bar{K}(t)$ denote $K(t)$ in (12) for $L(t)=I$. For this case, we have

$$
\begin{aligned}
\bar{K}(t) \bar{K}^{\prime}(t) & =\tilde{\Gamma}^{\prime}(t) Q_{t}^{1 / 2} H^{\prime}(t) H(t) Q_{t}^{1 / 2} \tilde{\Gamma}(t) \\
& =\tilde{\Gamma}^{\prime}(t) Q_{t}^{-1 / 2} Q_{t} H^{\prime}(t) H(t) Q_{t} Q_{t}^{-1 / 2} \tilde{\Gamma}(t) \\
& =\xi^{*}(t) \hat{\Gamma}^{\prime}(t) \bar{Q} \bar{H}^{\prime} \bar{H} \bar{Q} \hat{\Gamma}(t) \\
& =\xi^{*}(t) \hat{\Gamma}^{\prime}(t)\left\{A \bar{Q}+\bar{Q} A^{\prime}+G_{0}\right\} \hat{\Gamma}(t) \\
& =\hat{\Gamma}^{\prime}(t)\left\{A Q_{t}+Q_{t} A^{\prime}+\xi^{*}(t) G_{0}\right\} \hat{\Gamma}(t),
\end{aligned}
$$

where we have used the following relation for the last equality.

$$
\begin{aligned}
\xi^{*}(t) & \bar{Q} \hat{\Gamma}(t)=\xi^{*}(t) \bar{Q} Q_{t}^{-1 / 2} \tilde{\Gamma}(t)=\xi^{*}(t) \bar{Q} H^{\prime}(t) \bar{K}^{-1}(t) \\
& =\sqrt{\xi^{*}(t)} \bar{Q} \bar{H}^{\prime} \bar{K}^{-1}(t)=Q_{t} H^{\prime}(t) \bar{K}^{-1}(t) \\
& =Q_{t}^{1 / 2} \tilde{\Gamma}(t)=Q_{t} \hat{\Gamma}(t) .
\end{aligned}
$$

Note that the last expression of (58) does not depend on $H(t)$. By (58), we can write (53) as

$$
\begin{aligned}
\dot{\tilde{\Xi}}= & \xi^{*}(t)\left\{\bar{K}(t) \bar{K}^{\prime}(t)-K(t) K^{\prime}(t)\right\} \\
& +\xi^{*}(t)\left\{1-\xi^{*}(t)\right\} \hat{\Gamma}^{\prime}(t) G_{0} \hat{\Gamma}(t) .
\end{aligned}
$$

From (54) and (12), for a general value of $L(t)$, we have

$$
K(t)=\bar{K}(t) L(t) .
$$

Hence, we have

$$
\begin{aligned}
\dot{\tilde{\Xi}}= & \xi^{*}(t) \bar{K}(t)\left\{I-L(t) L^{\prime}(t)\right\} \bar{K}^{\prime}(t) \\
& +\xi^{*}(t)\left\{1-\xi^{*}(t)\right\} \hat{\Gamma}^{\prime}(t) G_{0} \hat{\Gamma}(t) .
\end{aligned}
$$

Note that $\operatorname{tr}\left[\bar{K}(t) \bar{K}^{\prime}(t)\right]=\operatorname{tr}\left[\bar{K}^{\prime}(t) \bar{K}(t)\right]=p \quad$ and $\operatorname{tr}\left[K(t) K^{\prime}(t)\right]=\operatorname{tr}\left[K^{\prime}(t) K(t)\right]=p$. Then, by taking trace of (60) and noting that (14) implies $\operatorname{tr}[\dot{\tilde{\Xi}}]=m \dot{\xi}^{*}(t)$, we have

$$
\dot{\xi}^{*}(t)=\frac{1}{m} \xi^{*}(t)\left\{1-\xi^{*}(t)\right\} \operatorname{tr}\left[\hat{\Gamma}^{\prime}(t) G_{0} \hat{\Gamma}(t)\right] .
$$

Thus, we have $\xi^{*}(t) \rightarrow 1$ as $t \rightarrow \infty$. Also, substitutions of $\dot{\tilde{\Xi}}=0$ and $\xi^{*}(t)=1$ into (62) yield $L(t) \rightarrow 1$ as $t \rightarrow \infty$ since $\operatorname{rank}[\bar{K}(t)]=m$. Consequently, we have $Q_{t} H^{\prime}(t) \rightarrow \bar{Q} \bar{H}^{\prime}$ and $H^{\prime}(t) \rightarrow \bar{H}^{\prime}$ as $t \rightarrow \infty$. Now, for $H^{\prime}(t)=\bar{H}^{\prime}$, it can be seen from (6) that

$$
\dot{Q}_{t}=A Q_{t}+Q_{t} A^{\prime}+G_{0}-Q_{t} \bar{H} \bar{H}^{\prime} Q_{t},
$$

from which we have $Q_{t} \rightarrow \bar{Q}$ by (21). This completes the proof. 


\section{An Algorithm to Compute Gain Matrix $H$}

In this section, we develop a recursive algorithm to compute $H(t)$ based on the results given in the previous section. Note that once we know the value of $Q_{t}$, we can easily compute $\xi^{*}(t)$ and $\tilde{\Gamma}(t)$. Hence, it is only necessary for us to determine $K(t)$ in (12) by (13) and (15). By taking sum of (15) with respect to $i$, we have

$$
\operatorname{tr}\left[K^{\prime}(t) K(t)\right]+\sum_{i=1}^{\ell} s_{i}(t)=\ell \tilde{\alpha}(t) .
$$

Hence, it can be seen from (13) that

$$
\tilde{\alpha}(t)=\frac{1}{\ell}\left[p(t)+\sum_{i=1}^{\ell} s_{i}(t)\right] .
$$

It should be noted that if we have $\tilde{\alpha}(t)-s_{i}(t)<0$, for any $i$ and $\tilde{\alpha}(t)$ given by (54), we cannot obtain $K(t)$ from (15). For this case, condition (15) is too strict and we should take

$$
\sum_{j=1}^{m}[K(t)]_{i j}^{2}=0 .
$$

for such $i$ and the value of $\tilde{\alpha}(t)$ should be modified from (15). We can easily obtain an algorithm to compute $K(t)$ including this modification of $\tilde{\alpha}(t)$ as follows.

\section{[A Recursive Algorithm to Compute $K(t)$ ]}

(Step 1) Let $k=0$ and $\Theta_{0} \triangleq\{1,2, \cdots, \ell\}$.

(Step 2) Compute

$$
\tilde{\alpha}_{k}(t)=\frac{1}{\sum_{i \in \Theta_{k}} 1}\left[p(t)+\sum_{i \in \Theta_{k}} s_{i}(t)\right],
$$

and let

$$
s^{*}(t) \triangleq \max _{i \in \Theta_{k}}\left\{s_{i}(t)\right\} .
$$

(Step 3) If $s^{*}(t)>\tilde{\alpha}_{k}(t)$, then define $\Theta_{k+1}$ by

$$
\Theta_{k+1} \triangleq \Theta_{k}-\left\{i \in \Theta_{k} ; s_{i}(t)=s^{*}(t)\right\},
$$

and repeat Step 2 with $k+1 \rightarrow k$. Otherwise, i.e., if we have $s^{*}(t) \leq \tilde{\alpha}_{k}(t)$, then proceed to Step 4 .

(Step 4) Set as $\tilde{\alpha}(t)=\tilde{\alpha}_{k}(t)$ and compute $K(t)$ by (15) for $i \in \Theta_{k}$ and

$$
[K(t)]_{i j}=0, \quad \text { for } 1 \leq j \leq m \text { and } i \notin \Theta_{k} .
$$

As we see, we can determine $K(t)$ by the above algorithm as far as there exists a solution.

\section{Concluding Remarks}

In this paper, we did not demonstrate numerical examples of the proposed method of the optimization of $H(t)$.
However, for $m=n$, numerical studies of the asymptotic property to the stationary solution are carried out in Takeuchi and Dei [10], [11]. Numerical examples for $m \leq n$ together with a consideration for numerical computation of LOOP will be reported in the near future.

\section{References}

[1] R. E. Kalman and R. S. Bucy: New results in linear filtering and prediction, Trans. ASME, J. Basic Eng. 82 D, pp.95-108, 1960.

[2] M. Athans, On the determination of optimal costly measurement strategies for linear stochastic systems, Prep. 5th IFAC World Congress, Paris, 1/11, 1972.

[3] M. Aoki and M. T. Li, Optimal discrete time control system with cost for observation, IEEE Trans. Automatic Control, AC-14, 2, 165/175, 1969.

[4] A. Sano and M. Terao, Measurement optimization in optimal process control, Automatica, 5, 705/714, 1970.

[5] A. Logothetis, A. Isaksson and E. Skafidas, On sensor scheduling via information theoretic criteria, Prep. ACC, 1999.

[6] Y. Takeuchi and T. Ishikawa, The optimal transmission of a set of Gaussian signals through parallel channels with feedback, Proc. of 25th ISCIE SSS, Osaka, Japan, Nov. 10-12, 255/260, 1993.

[7] Y. Takeuchi, The optimal transmission of correlated Gaussian signals through parallel channels with feedback, Proc. of IEEE 35th CDC, Kobe, Japan, 2 , 1890/1895, 1996.

[8] Y. Takeuchi and M. Sowa, An information theoretic approach to sensor allocation of Kalman-Bucy filter, Proc. of 33rd ISCIE SSS, Tochigi, Japan, Oct. 29-30, 2001, 234/239, 2002.

[9] Y. Takeuchi, M. Sowa and H. Horikawa, An information theoretic scheme for sensor allocation of linear least-squares estimation, Proc. of SICE Annual Conference in Osaka, Aug. 5-7, 2002, 248/253, 2002.

[10] Y. Takeuchi and K. Dei, The optimal transmission of a set of Gaussian signals through parallel channels with feedback, Proc. of 34th ISCIE SSS, Fukuoka, Japan, Oct. 31-Nov. 1, 2002, 78/83, 2003.

[11] Y. Takeuchi and K. Dei, The optimal transmission of Nonstationary Gaussian signals through parallel channels with feedback, Proc. of SICE Annual Conference in Fukui, Aug. 4-6, 2003, 2901/2906, 2003.

[12] Y. Takeuchi, "The optimal transmission of nonGaussian signals through a noisy channel with feedback," IEEE Trans. Inform. Theory, IT-40, 5, 1624/1629, 1994. 